\title{
KamLAND status and results
}

\section{Jean-Stephane Ricol ${ }^{* \dagger}$}

RCNS, Tohoku University

E-mail: ricoleawa.tohoku.ac.jp

The recent KamLAND results confirm the reactor antineutrinos disappearence at $99.998 \% \mathrm{CL}$ and show significant spectrum distorsions that give further support for the conclusion of neutrino oscillation. A two-neutrino analysis of KamLAND data leads to a precise determination of the parameter $\Delta m^{2}=7.9_{-0.5}^{+0.6} \times 10^{-5} \mathrm{eV}^{2}$. Assuming CPT invariance, a global analysis of data from KamLAND and solar experiments yields $\Delta m^{2}=7.9_{-0.5}^{+0.6} \times 10^{-5} \mathrm{eV}^{2}$ and $\tan ^{2} \theta=0.40_{-0.07}^{+0.10}$.

KamLAND has also the sensitivity to detect geologically produced antineutrinos and results give a first positive signature. Assuming a $\mathrm{Th} / \mathrm{U}$ concentration ratio of 3.9 the $90 \%$ confidence interval for the total number of genoneutrinos is 4.5 to 54.2 .

The collaboration is now preparing the purification of the detector for the detection of ${ }^{7} \mathrm{Be}$ solar neutrinos.

International Europhysics Conference on High Energy Physics

July 21st - 27th 2005

Lisboa, Portugal

*Speaker.

$\dagger$ on behalf of KamLAND collaboration 


\section{Introduction}

The main goal of KamLAND 1000 tons liquid scintillator detector is the study of neutrino oscillation through the detection of reactor electronic antineutrinos. The 766 ton-year exposure results [1] confi rmed the antineutrinos disappearance at 99.998\% CL. With the precise measurement of the events energy, KamLAND gives a precise measurement of $\Delta m^{2}$ while $\sin ^{2} \theta$ is constrained by solar experiments.

The low threshold and low background of the detector allow for the detection of geologically produced antineutrinos, which are an unique way to learn about Earth internal composition and radiogenic power. Recent results showed the fi rst positive signature of these geoneutrinos [2] that is consistent with Earth models.

KamLAND collaboration is now working on the purifi cation of the detector in order to measure in real time the flow of solar ${ }^{7} \mathrm{Be}$ neutrinos with an expected precision of $10 \%$, that will improve the current precision by a factor 4 .

\section{KamLAND experiment}

The KamLAND detector consists of $1 \mathrm{kton}$ of ultra-pure liquid scintillator (LS) surrounded by an array of 1879 photomultiplier tubes. Electronic antineutrinos are detected via the inverse beta decay reaction : $\overline{\mathrm{v}}_{e}+p \rightarrow e^{+}+n$. The prompt scintillation light of the $e^{+}$gives an estimate of the incident $\bar{v}_{e}$ energy and the $\sim 200 \mu$ s delayed $2.2 \mathrm{MeV} \gamma$-ray from neutron capture on hydrogen is a powerful tool to reduce backgrounds. KamLAND calibration is made by deploying $\gamma$-ray and neutron sources along the vertical axis. Trace contaminants on the ballon and within the LS are also exploited. The energy resolution is $6.2 \% \sqrt{E(M e V)}$. The fi ducial volume is defi ned by the cuts on the radial position and we assess its systematic incertainty by studying the uniformlydistributed muon spalation products. Selection cuts are based on time and spatial coincidence between prompt and delayed event and energy cuts. We also apply a veto after muon. Remaining backgrounds come from accidental coincidence, spalation long-lived delayed-neutron $\beta$-emitters $\left({ }^{9} \mathrm{Li}\right.$ and $\left.{ }^{8} \mathrm{He}\right)$ and ${ }^{13} \mathrm{C}(\alpha, n){ }^{16} \mathrm{O}$ reaction where $\alpha$ come from the ${ }^{210} \mathrm{Po}$ decay. More details about analysis, backgrounds, selection cuts and systematic errors can be found in [1] and [2].

\section{Reactor $\bar{v}_{e}$ results}

Data presented here are based on a 766.3 tons-year exposure of KamLAND to reactor antineutrinos. Most of antineutrinos $(\sim 80 \%)$ are produced at a baseline of $180 \pm 35 \mathrm{~km}$. In the absence of neutrino oscillation, we expect $365 \pm 23.7$ antineutrinos and $17.8 \pm 7.3$ background events above 2.6 MeV. KamLAND observed 258 events, confi rming $\bar{v}_{e}$ disappearance at the $99.998 \%$ signifi cance level. Signifi cant variations in antineutrinos flux due to several reactors maintenance give the possibility to check that the signal is consistent with reactor antineutrinos and known background. The best fi t spectrum together with the background is shown in Figure. 1 and gives $\Delta m^{2}=7.9_{-0.5}^{+0.6}$ $\mathrm{eV}^{2}$ and $\tan ^{2} \theta=0.46$. Combined with solar experiments, KamLAND results lead to $\Delta m^{2}=7.9_{-0.5}^{+0.6}$ $\mathrm{eV}^{2}$ and $\tan ^{2} \theta=0.46_{-0.07}^{+0.10}$. 


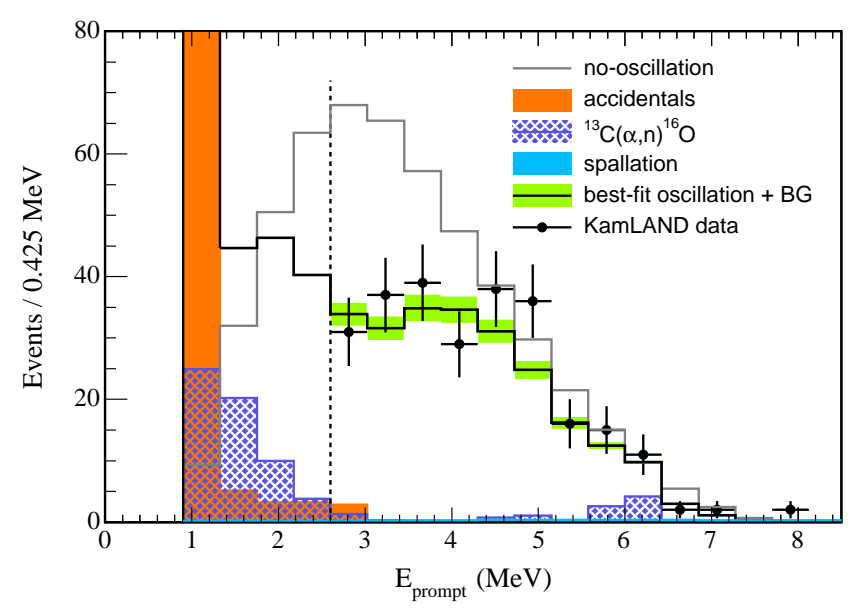

Figure 1: Prompt event energy spectrum of $\bar{v}_{e}$ candidates and associated background.

\section{Geo-neutrinos results}

The detection of geologically produced neutrinos is an unique opportunity to obtain informations about Earth internal composition and energy generation mechanism. KamLAND has the sensitivity to detect antineutrinos produced in ${ }^{238} \mathrm{U}$ and ${ }^{232} \mathrm{Th}$ decay chains for which Earth composition models suggest a radiogenic power of $16 \mathrm{TW}$ (half of the total measured heat dissipation rate).

The detection of these geo-antineutrinos [2] is the same as for reactor ones with tighter selection cuts and different energy window. Background for geoneutrinos is dominated by reactor $\overline{\mathrm{v}}_{e} \mathrm{~s}$ (80.4 events) and by $13 C(\alpha, n){ }^{16} O$ reactions (42 events) due to radioactive contamination in the detector. There is a small contribution from accidental coincidences and long lived nuclear reactor fi ssion products. The total background is estimated to be $127 \pm 13$ events.

The total number of observed $\bar{v}_{e}$ candidates is 152 , with their energy distribution shown in Figure.2. A 'rate only' analysis gives $25_{-18}^{+19}$ geoneutrino candidates which corresponds to a rate of $5.1_{-3.6}^{+3.9} \times 10^{-31} \bar{v}_{e}$ per target proton per year.

Based on a study of chondritic meteorites, the $\mathrm{Th} / \mathrm{U}$ mass ratio is believed to be between 3.7 and 4.1 and is known better than either absolute concentration. Assuming a $\mathrm{Th} / \mathrm{U}$ mass ratio of 3.9, an un-binned likelihood analysis yields to a total number of geoneutrinos between 4.5 and 54.2 at $90 \%$ confi dent level, with a central value of 28.0 events.

\section{Solar phase}

So far only a very low percentage of total solar neutrinos have been measured in real time. KamLAND next goal is the detection of solar $v_{e}\left({ }^{7} \mathrm{Be}\right)$ in order to improve the current $40 \%$ incertainty on the flux by a factor 4 . This purpose requires an intense purifi cation of low energy background in the detector. The main contaminants are ${ }^{210} \mathrm{~Pb},{ }^{85} \mathrm{Kr},{ }^{39} \mathrm{Ar}$ and ${ }^{40} \mathrm{~K}$ and a large effort of research and development using distillation technique and noble gaz purge tower show very encouraging preliminary results. The purifi cation system will be constructed in 2006. 


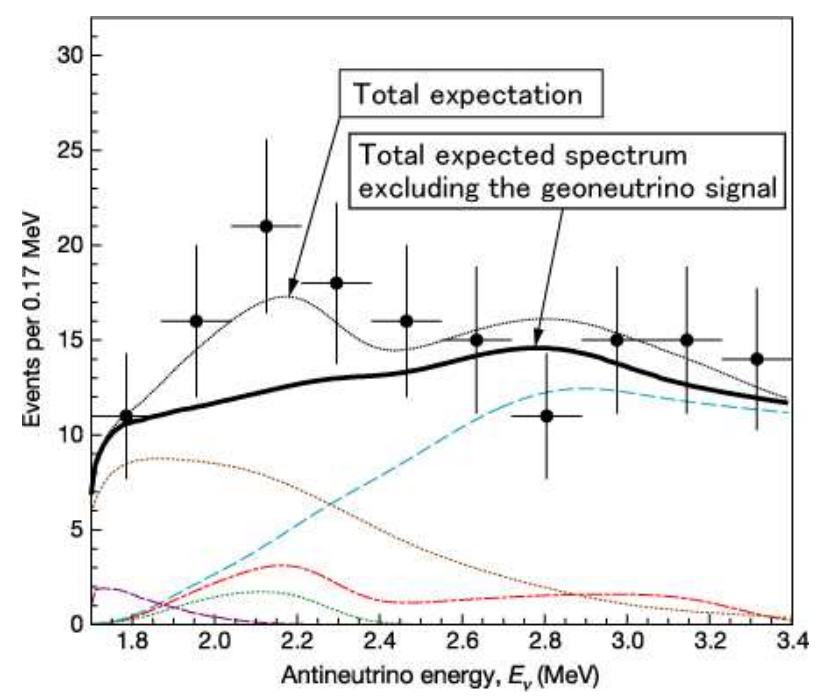

Figure 2: $\overline{\mathrm{v}}_{e}$ energy spectra: experimental points with total expectation. Also shown are the total expected spectrum excluding geo-neutrinos, the expected signal from ${ }^{238} \mathrm{U}$ (dot-dashed red line) and ${ }^{232} \mathrm{Th}$ (dotted green line) geoneutrinos, and the backgrounds due to reactor $\bar{v}_{e}$ (dashed light blue line), ${ }^{13} C(\alpha, n){ }^{16} O$ reactions (dotted brown line), and random coincidence (dashed purple line).

\section{Conclusion}

KamLAND results show reactor antineutrino disappearence at $99.998 \%$ CL and spectral distorsion at $99.6 \% \mathrm{CL}$. We recently entered an era of precise measurement, we now have only one permitted solution to the oscillations parameters.

KamLAND made the fi rst experimental investigation of geoneutrinos opening a new research fi eld in neutrino physics and is now on its way to measure ${ }^{7} \mathrm{Be}$ solar neutrinos.

\section{Acknowledgments}

The KamLAND experiment is supported by the COE program of the Japanese Ministry of Education, Culture, Sports, Science and Technology, and by the United States Department of Energy. The reactor data were provided by courtesy of the following electric associations in Japan : Hokkaido, Tohoku, Tokyo, Hokuriku, Chubu, Kansai, Chugoku, Shikoku and Kyushu Electric Power Companies, Japan Atomic Power Co. and Japan Nuclear Cycle Development Institute. Mining and Smelting Company has provided service for activities at the experimental site.

\section{References}

[1] T. Araki et al. (KamLAND collaboration) Phys. Rev. Lett. 94, 081801,2005.

[2] T. Araki et al. (KamLAND collaboration) Nature 436, 2005. 